\title{
Implementation of tranexamic acid for bleeding trauma patients: a longitudinal and cross- sectional study
}

\author{
Timothy J Coats, ${ }^{1}$ Marisol Fragoso-Iñiguez, ${ }^{2}$ lan Roberts ${ }^{3}$
}

\begin{abstract}
${ }^{1}$ Emergency Medicine, University of Leicester, Leicester, UK ${ }^{2}$ Trauma Audit and Research Network (TARN), University of Manchester Institute of Population Health, Manchester, UK

${ }^{3}$ Nutrition and Public Health Intervention Research Unit, London School of Hygiene and Tropical Medicine, London, UK
\end{abstract}

\section{Correspondence to}

Dr Timothy I Coats, University of Leicester, Leicester LE1 7RH, UK; tc61@le.ac.uk

Received 4 April 2018 Revised 9 November 2018 Accepted 20 November 2018 Published Online First 8 December 2018

\section{Check for updates}

(C) Author(s) (or their employer(s)) 2019. No commercial re-use. See rights and permissions. Published by BMJ.

To cite: Coats TJ,

Fragoso-Iñiguez M

Roberts I. Emerg Med J

2019:36:78-81.

\section{ABSTRACT}

Objective To describe the use of tranexamic acid (TXA) in trauma care in England and Wales since the Clinical Randomization of an Antifibrinolytic in Significant Hemorrhage (CRASH-2) trial results were published in 2010.

Methods A national longitudinal and cross-sectional study using data collected through the Trauma Audit and Research Network (TARN), the clinical audit of major trauma care for England and Wales. All patients in the TARN database injured in England and Wales were included apart from those with an isolated traumatic brain injury, with a primary outcome of the proportion of patients given TXA and the secondary outcome of time to treatment.

Results Among 228250 patients, the proportion of trauma patients treated with TXA increased from near zero in 2010 to $10 \%$ (4593) in 2016. In 2016, most patients (82\%) who received TXA did so within 3 hours of injury, however, only $30 \%$ of patients received TXA within an hour of injury. Most (80\%) of the patients who had an early blood transfusion were given TXA. Patients treated with TXA by an ambulance paramedic received treatment at a median of 49 min (IQR 33-72) compared with $111 \mathrm{~min}$ (IQR 77-162) for patients treated in hospital.

Conclusions There is a low proportion of patients treated with TXA across the range of injury severity and the range of physiological indicators of severity of bleeding. Most patients receive treatment within the existing target of 3 hours from injury, however there remains the potential to further improve major trauma outcomes by the earlier treatment of a wider patient group.

\section{INTRODUCTION}

The Clinical Randomization of an Antifibrinolytic in Significant Hemorrhage 2 (CRASH-2) trial of tranexamic acid (TXA) in 20210 bleeding trauma patients was published in 2010 and showed that this inexpensive and widely practicable treatment safely reduces death due to bleeding and all-cause mortality. ${ }^{1}$ Subsequent prespecified subgroup analyses published in 2011 showed that the effect depends importantly on the time interval between injury and the start of treatment. ${ }^{2}$ Treatment given within 3 hours of injury substantially reduces death due to bleeding, whereas treatment initiated after 3 hours is ineffective. ${ }^{3}$ The survival benefits from TXA do not appear to vary by baseline risk of death, suggesting that it can be used in a wide spectrum of

\section{Key messages}

What is aready know on this subject

- Tranexamic acid reduces mortality from bleeding after major trauma.

What this study adds

- Tranexamic use increased from 0\% in 2010 to $10 \%$ of patients in the TARN database by 2014 .

- Only $30 \%$ of treated patients receive TXA within 1 hour of injury.

- Many patients who have physiological markers of bleeding or injury severity scores do not receive TXA.

patients and that its use should not be restricted to the most severely injured. ${ }^{4}$

In response to these results, TXA was included in the WHO list of essential medicines and in clinical guidelines for the management of acute traumatic bleeding. ${ }^{5}$ The entry criteria for the CRASH-2 trial were 'adult trauma patients with or at risk of significant haemorrhage.' These simple criteria allowed participating doctors to use their clinical judgement when deciding whether patients were at risk of death from bleeding, just as they would in normal medical practice. Worldwide, clinical guidelines have also taken this approach and recommended that TXA be administered as soon as possible to any trauma patient with or at risk of significant haemorrhage. In England, the NHS has a system, the best practice tariff, in which effective patient care is incentivised by an additional per patient payment (in 2017 of $£ 2800$, US\$3700). The payments are dependent on achieving a series of quality standards, which in the case of trauma care, are laid out on the 'Trauma Dashboard'. The administration of TXA to severely injured patients is one of these standards. As the number of patients 'with or at risk of significant bleeding' is not routinely recorded in the NHS, a proxy measure had to be used to monitor compliance with the standard. The additional payment is given if a trauma patient who received blood or blood products within 6 hours of admission also received TXA within 3 hours of injury.

Recent research has underscored the importance of early TXA treatment. An individual patient data meta-analysis that included over 40000 patients with acute severe bleeding has shown that immediate TXA treatment improves survival by $70 \%$, 
Table 1 Data fields and data definitions

\begin{tabular}{|c|c|}
\hline Time of injury & $\begin{array}{l}\text { First time recorded —either time of injury or time } \\
\text { of initial emergency call }\end{array}$ \\
\hline BP & First recorded systolic BP in hospital \\
\hline Pulse rate & First recorded pulse rate in hospital \\
\hline RR & First recorded RR in hospital \\
\hline TXA given & TXA use-yes/no \\
\hline Location of TXA given & $\begin{array}{l}\text { Location of patient when TXA was given- } \\
\text { prehospital, ED or other }\end{array}$ \\
\hline Time that TXA was given & Time of start of TXA infusion \\
\hline Probability of survival (Ps) & Ps calculated by the standard TARN method ${ }^{8}$ \\
\hline $\begin{array}{l}\text { Use of blood products within } \\
6 \text { hours }\end{array}$ & Any products transfused-yes/no \\
\hline
\end{tabular}

TARN, Trauma Audit and Research Network; TXA, tranexamic acid.

but thereafter the survival benefit decreases by about $10 \%$ for every 15 min treatment delay until 3 hours, after which there is no survival benefit. ${ }^{6}$

We used data from the UK Trauma Audit and Research Network (TARN) to describe the implementation of the CRASH-2 trial results into clinical practice in England and Wales.

\section{METHODS}

The TARN was established in England and Wales from 1990 to measure and monitor the process of trauma care and the outcomes for patients. Trauma patients are included in the TARN database if they have either a hospital admission of $>72$ hours due to injury, or a critical care admission, or transfer to a tertiary/specialist centre or death within 30 days. Patients with isolated femoral neck or single pubic ramus fracture who are older than 65 years and those with simple isolated injuries are excluded. In 2010, the use of TXA was included as an additional mandatory data field. The UK Health Research Authority Patient Information Advisory Group has given approval (Section 20) for research using anonymised TARN data.

To describe the use of TXA, the variables shown in table 1 were extracted for all patients fitting TARN inclusion criteria from 2010 to 2016 (database interrogated in November 2017). Patients were excluded from the analysis if they had an Injury Severity Score $<9$, an isolated head injury (defined as an

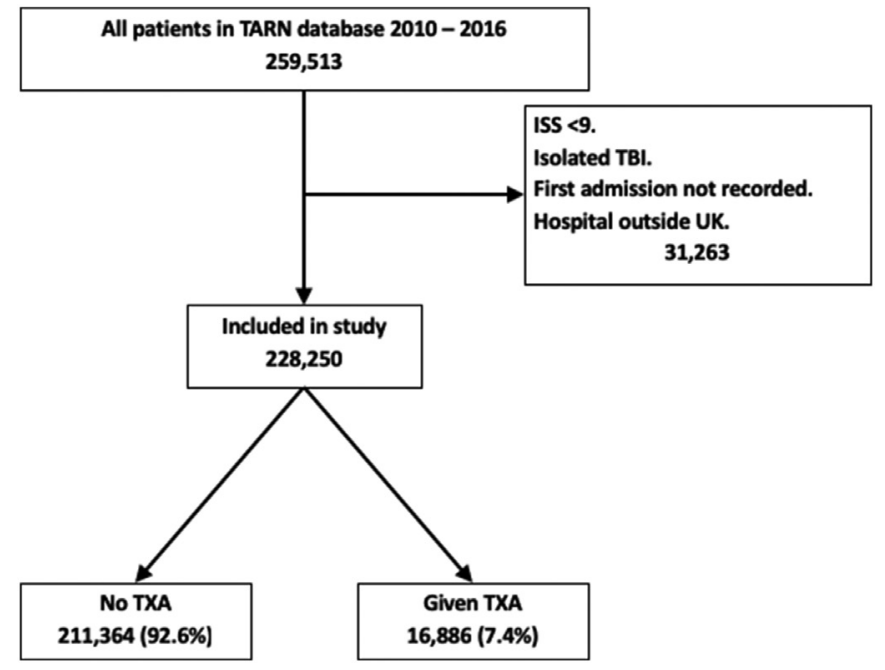

Figure 1 Diagram of included patients. ISS, Injury Severity Score; TARN, Trauma Audit and Research Network; TBI, traumatic brain injury; TXA, tranexamic acid.

\begin{tabular}{lcl} 
Table 2 & Missing data for physiology variables & \\
\hline Missing parameter & $\begin{array}{l}\text { Not given TXA } \\
(\mathbf{n}=\mathbf{2 1 1 3 6 4 ) , \%}\end{array}$ & $\begin{array}{l}\text { Given } \\
\text { TXA }(\mathbf{n}=16886), \%\end{array}$ \\
\hline Systolic BP & $16654(7.9)$ & $1020(6.0)$ \\
RR & $24233(11.5)$ & $3752(22.2)$ \\
Pulse rate & $14137(6.7)$ & $650(3.8)$ \\
GCS & $7238(3.4)$ & $231(1.4)$ \\
\hline
\end{tabular}

TXA, tranexamic acid.

Abbreviated Injury Score ${ }^{7}$ (AIS) of 3 or more in the head and no other AIS greater than 2), had an interhospital transfer without the first admission being recorded, or who were submitted from a hospital outside of the UK.

We used descriptive statistics to illustrate the change in use of TXA over time from 2010 to 2016, the relationship between TXA use and injury severity (based on probability of survival (Ps) and physiology), the time to treatment and the location of treatment.

\section{RESULTS}

There were 228250 patients in the TARN database who fulfilled the study criteria (figure 1). The proportions of missing data for each physiological parameter are shown in table 2-these patients were excluded from the corresponding subanalysis of the relationship between TXA use and physiology. There were 6723 patients $(2.9 \%)$ who did not have sufficient data for a $\mathrm{Ps}^{8}$ to be calculated. These patients were excluded from the subanalysis of the relationship between TXA use and Ps.

The annual number of patients fulfilling the study criteria increased during the study period from 16554 in 2010 to 45850 in 2016. The improvement in national data collection between 2010 and 2016 coincided with the implementation of a 'trauma network' system across England and Wales, ${ }^{9}$ leading to a large increase in the number of hospitals contributing to the database.

The proportion of patients treated with TXA rapidly increased from 2011 (figure 2), levelling off after 2014. However, by the end of the study period TXA was given to only $10 \%$ of patients with injuries severe enough to be included in the national major trauma database. The plot of TXA use against Ps (figure 3) shows a weak upward trend, but even the most severely injured patients had a low (20\%) probability of TXA treatment.

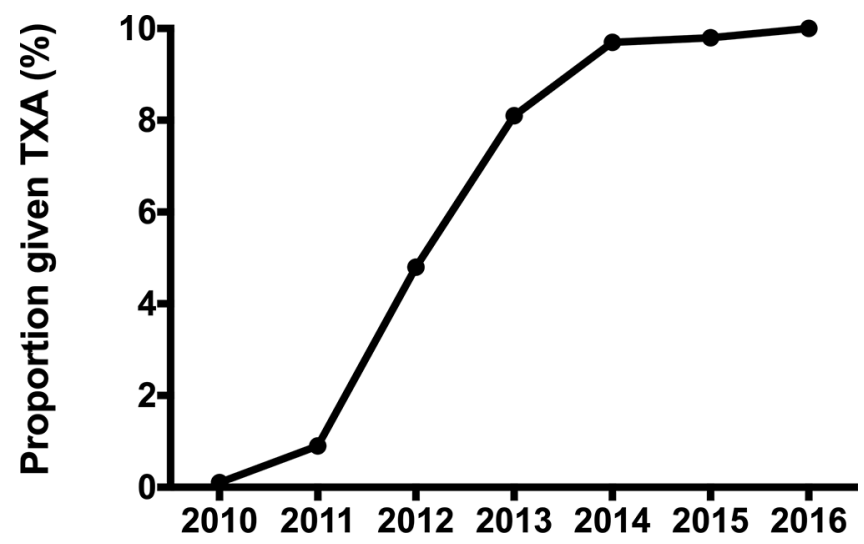

Figure 2 Proportion of patients receiving tranexamic acid (TXA) by year. 


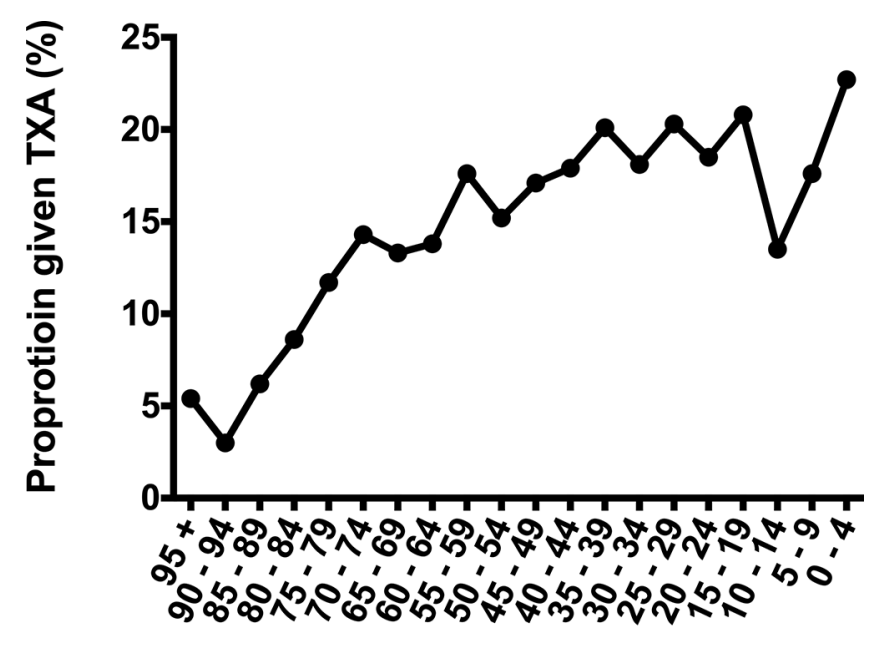

Probability of Survival (\%)

Figure 3 Proportion given tranexamic acid (TXA) by probability of survival.

A similar pattern was seen with physiological severity of injury, where the proportion of patients given TXA was low, even in those with a clinical indication that they might have significant bleeding (table 3$)$, such as a low BP $(23.8 \%$ treated with TXA at systolic $\mathrm{BP}<100 \mathrm{~mm} \mathrm{Hg}$ ) or high pulse rate $(23.9 \%$ given TXA at a pulse rate of $>140 \mathrm{bpm})$. However, of the patients who were bleeding sufficiently to require a transfusion of blood products during the first 6 hours $69 \%$ were transfused (increasing from $0 \%$ in 2010 to $80 \%$ in 2016).

The median time to treatment with TXA in 2016 was 1.45 hours (IQR $0.85-2.50$ ) with little variation over the study period. Most patients (82\%) received TXA within the 3 hours of injury, however only $30 \%$ of patients received TXA within the first hour (figure 4). In patients who had a transfusion within 6 hours, the median time to treatment was 1.22 hours (IQR 0.75-1.97). Time to treatment was shorter for patients treated by ambulance service personnel (median $49 \mathrm{~min}$, IQR 33-72) than for patients treated in hospital (median $111 \mathrm{~min}, \mathrm{IQR}$ 77-162), however this might be confounded by ease of diagnosis of bleeding and simply represent early treatment of patients with obvious bleeding.

Table 3 The relationship of TXA use with physiology (2010-2016)

\begin{tabular}{lc}
\hline & Proportion of all patients given TXA, \% \\
\hline BP & \\
SBP $<100$ & 23.8 \\
SBP $\geq 100$ & 6.4 \\
\hline$R R$ & 10.9 \\
$0-13$ & 4.6 \\
$14-20$ & 12.1 \\
$21-30$ & 17.1 \\
$31-40$ & 12.7 \\
$>40$ & \\
\hline Pulse rate & 5.7 \\
$<100$ & 12.7 \\
$100-120$ & 22.5 \\
$121-140$ & 23.9 \\
\hline 140 & \\
\hline
\end{tabular}

SBP, systolic BP; TXA, tranexamic acid.

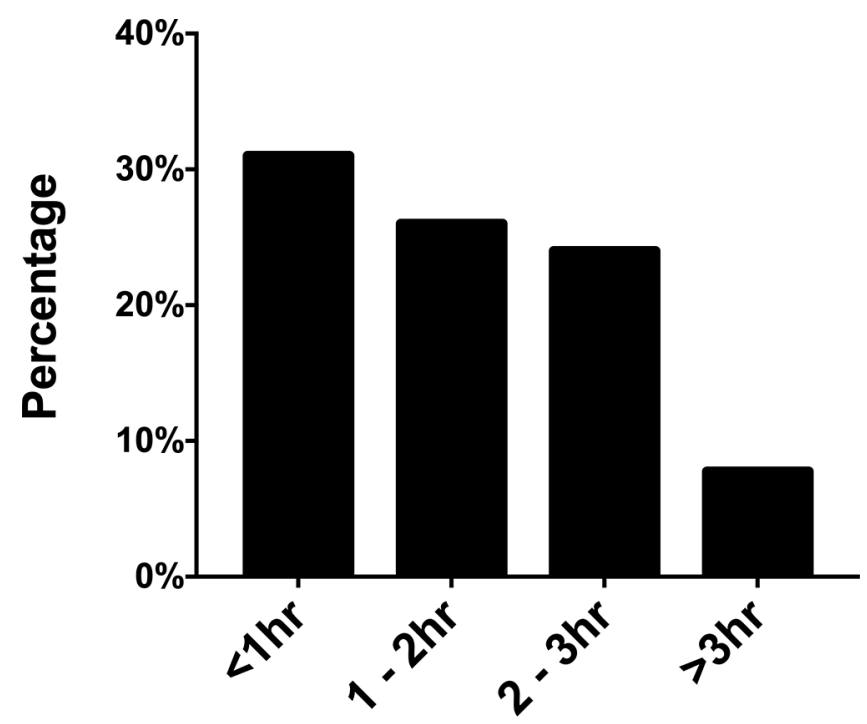

Time from Injury to Treatment

Figure 4 Time to tranexamic acid (TXA) treatment.

\section{DISCUSSION}

The analysis shows a low level of TXA use in England and Wales, even in groups with early physiological abnormalities that could indicate significant haemorrhage, or with severe injury. The only subgroup with a high proportion of patients treated was those who required an early transfusion. This could be interpreted as correct use of the drug (as it is being given to the patients who need transfusion), however the indication for TXA use, derived from the CRASH-2 trial results, is the treating clinician's estimate of baseline risk of bleeding, not the need for transfusion. The low overall treatment rate in major trauma patients suggests that many patients who could potentially benefit from TXA are not being treated.

The data suggest that the use of TXA in trauma patients seems to be associated with the receipt of blood transfusion rather than by the initial clinical state (injury severity or physiological abnormality). Clinicians may be following 'massive transfusion' guidance that applies to all major haemorrhage ${ }^{10}$ rather than specifically to trauma bleeding. This may have led to TXA being incorrectly thought of as part of the 'massive transfusion policy' rather than as a drug for the early trauma resuscitation phase.

The mechanism of action of TXA in preventing death after major trauma is not fully understood. It seems from our data that clinicians are assuming that it stabilises clot in patients who have severe bleeding, and in fact some have advocated not using TXA unless there is demonstrable hyperfibrinolysis. ${ }^{11}$ However, there is little evidence for a belief that the drug only works in severe bleeding (about half of the patients in the CRASH-2 trial did not receive a blood transfusion), as the available data suggest that the benefit of TXA does not vary by baseline risk of death in patients who have a clinical suspicion of significant bleeding. ${ }^{4}$

The data presented on the current time to treatment also suggest that TXA is not being used in the most effective way. Across a range of bleeding conditions tranexamic treatment is extremely time dependent, with $10 \%$ of the benefit being lost for every 15 min delay. ' 'High quality' treatment has been defined within UK trauma systems as starting the TXA infusion within 3 hours of injury, however it is clear that patients in the later stages of this time window get little or no benefit. There are still many patients getting TXA in the second or third hour after 
injury (which may coincide with the point at which a decision for transfusion has been made).

Our study has several limitations. The criteria for TXA administration (the patient is bleeding or at significant risk of bleeding) are seldom documented in patient notes and so proxy measures (blood transfusion, injury severity or physiology) have to be used to assess 'appropriateness' of care. The relevance of these proxy measures can be challenged (eg, is blood transfusion within 6 hours a good proxy measure of need for TXA treatment when half of the patients in the CRASH-3 trial did not receive blood products?). There is the need to develop more objective criteria for TXA treatment, based on prognostic models and including some consensus about the threshold level of risk based on cost-effectiveness considerations.

There are additional limitations within the TARN data collection system, as case ascertainment is much better at major trauma centres than trauma units, when compared with centrally collected statistics (Hospital Episode Statistics), which might lead to overall TXA use being overestimated if trauma centres are better at giving TXA. There was also a large change in the overall case ascertainment during the study period, with the total number of patients recorded more than doubling. It is likely that the population has changed, in particular with the discovery of more elderly major trauma patients within smaller hospitals who had previously been invisible to the national trauma audit system. However, it seems unlikely that the increase in TXA use observed in our study is due to differences in case ascertainment, since the inclusion of patients from smaller non-specialist hospitals would be expected to reduce rather than inflate the level of TXA use. Although we suspect that need for transfusion is driving the use of TXA, we did not have any data on the relative timing of TXA administration and the decision to transfuse. A further limitation is that we could not measure potential adverse consequences of the introduction of TXA into UK trauma care. For example, it is possible, but unlikely, that in spending time in the resuscitation phase giving TXA clinicians might have paid less attention to other aspects of patient treatment.

To most effectively decrease overall trauma mortality TXA must be given early ${ }^{6}$ in all patients suspected of having significant bleeding ${ }^{1}$ (as per the CRASH-2 inclusion criteria). Early treatment is not currently being achieved, except in those patients treated during the prehospital phase, perhaps suggesting that TXA should be more widely implemented as a prehospital drug. A change in clinical approach may be required to consider TXA treatment as a Primary Survey-Critical Intervention for suspect bleeding under the ' $\mathrm{C}$ ' of the $\mathrm{ABC}$ of the trauma primary survey. This would also have the effect of breaking the erroneous link that seems to have been made between TXA treatment and blood transfusion. It could be argued that whenever an intravenous cannula is inserted in a potentially severely injured patient, tranexamic acid should be given before considering any other fluid therapy.

Contributors The article was conceived and initially drafted by TJC and IR. All authors contributed to commenting on drafts of the manuscript and were involved in each stage of planning, interpretation of results and write-up of the work. The statistical analysis was performed by MFI. The final version has been approved by all authors.

Funding The Trauma Audit and Research Network (TARN) data collection is funded by the National Health Service of England and Wales through subscriptions from participating hospitals.

Competing interests TJC and IR were investigators for the CRASH-2 trial; no other relationships or activities could appear to have influenced the submitted work. TARN is based within a university department and is funded through subscriptions from the hospitals that take part in the audit.

Patient consent for publication Not required.

Provenance and peer review Not commissioned; externally peer reviewed.

Data sharing statement Patient-level data are available, subject to a standard data sharing agreement (which can be found at www.tarn.ac.uk), from the corresponding author at tc61@le.ac.uk. Individual participant consent for data sharing was not obtained, but the presented data are grouped and anonymised, risk of identification is low and approval is in place from the UK Department of Health's Patient Information Advisory Group.

\section{REFERENCES}

1 Shakur H, Roberts I, Bautista R, et al. Effects of tranexamic acid on death, vascular occlusive events, and blood transfusion in trauma patients with significant haemorrhage (CRASH-2): a randomised, placebo-controlled trial. Lancet 2010;376:23-32.

2 Roberts I, Shakur $\mathrm{H}$, Afolabi A, et al. The importance of early treatment with tranexamic acid in bleeding trauma patients: an exploratory analysis of the CRASH-2 randomised controlled trial. Lancet 2011;377:1096-101.

3 Roberts I, Edwards P, Prieto D, et al. Tranexamic acid in bleeding trauma patients: an exploration of benefits and harms. Trials 2017;18:48.

4 Roberts I, Perel P, Prieto-Merino D, et al. Effect of tranexamic acid on mortality in patients with traumatic bleeding: prespecified analysis of data from randomised controlled trial. BMJ 2012;345:e5839.

5 Spahn DR, Bouillon B, Cerny V, et al. Management of bleeding and coagulopathy following major trauma: an updated European guideline. Crit Care 2013;17:R76.

6 Gayet-Ageron A, Prieto-Merino D, Ker K, et al. Effect of treatment delay on the effectiveness and safety of antifibrinolytics in acute severe haemorrhage: a metaanalysis of individual patient-level data from 40138 bleeding patients. The Lancet 2017.

7 Committee on Medical Aspects of Automotive Safety. Rating the severity of tissue damage. I. The abbreviated scale. JAMA 1971;215:277-80.

8 Bouamra 0 , Jacques $\mathrm{R}$, Edwards $\mathrm{A}$, et al. Prediction modelling for trauma using comorbidity and 'true' 30-day outcome. Emerg Med J 2015;32:933-8.

9 McCullough AL, Haycock JC, Forward DP, et al. II. Major trauma networks in England. Br J Anaesth 2014;113:202-6.

10 Hunt BJ, Allard S, Keeling D, et al. A practical guideline for the haematological management of major haemorrhage. Br J Haematol 2015;170:788-803.

11 Napolitano LM, Cohen MJ, Cotton BA, et al. Tranexamic acid in trauma: how should we use it? J Trauma Acute Care Surg 2013;74:1575-86. 